\title{
Cinética y Mecanismo de la descomposición Térmica del Nipecotato de Etilo e Isonipecotato de Etilo
}

\author{
Amelia A. Espitia y Jennifer J. Lafont ${ }^{*}$ \\ Departamento de Química, laboratorio de Cinética y biocombustibles, Universidad de Córdoba, \\ Cra 6 No 76-103, Montería-Colombia. (e-mail: jenniferlafontmendoza@gmail.com; \\ andreaespitia36@gmail.com) \\ * autor a quien debe ser dirigida la correspondencia
}

Recibido Abr. 19, 2013; Aceptado May. 29, 2013; Versión final recibida Jul. 26, 2013

\begin{abstract}
Resumen
El objetivo de este estudio fue realizar la termólisis de dos compuestos aún no reportados en la literatura: nipecotato de etilo e isonipecotato de etilo; con el fin de encontrar las constantes de velocidad de degradación térmica, los parámetros de activación y proponer un mecanismo de reacción. Las termólisis fueron realizadas en un minireactor químico a cinco temperaturas entre $523 \mathrm{~K}$ y $563 \mathrm{~K}$ con diferencia de diez grados, los valores de las áreas fueron medidos con un cromatógrafo de gases acoplado a espectrometría de masas, usados también en la identificación de los productos de cada reacción. Se encontró que el nipecotato de etilo se degradó más rápidamente que el isonipecotato de etilo y en ambas reacciones las constantes de velocidad aumentaron con la temperatura y demostraron ser unimoleculares y de primer orden. Se propone un mecanismo con estado de transición cíclico, de seis miembros concertado y semipolar.
\end{abstract}

Palabras clave: cinética, mecanismo de reacción, descomposición térmica, nipecotato de etilo, isonipecotato de etilo

\section{Kinetics and Mechanism of the Thermal Decomposition of Ethyl Nipecotate and Ethyl Isonipecotate}

\begin{abstract}
The objective of this study was to carry out the thermolysis of two compounds not yet reported in literature: ethyl nipecotate and ethyl isonipecotate, in order to find the thermal degradation rate constants and the activation parameters and to propose a reaction mechanism. The thermolysis experiments were performed in a chemical minireactor at five temperatures between $523 \mathrm{~K}$ and $563 \mathrm{~K}$ with ten degrees difference; the values of the areas were measured with a gas chromatography coupled to mass spectrometry, also used in the identification of products of each reaction. It was found that ethyl nipecotate was degraded faster than ethyl isonipecotate and in both reactions the rate constants increased with the temperature and demonstrated to be unimolecular and of first order. A mechanism is proposed with a concerted, semi- polar six- member cyclic transition state.
\end{abstract}

Keywords: kinetics, reaction mechanism, thermal decomposition, ethyl nipecotate, ethyl isonipecotate. 


\section{INTRODUCCIÓN}

Los compuestos nipecotato de etilo e isonipecotato de etilo son ésteres etílicos de ácidos piperidincarboxílicos, cuyo nitrógeno está ubicado en las posiciones 3 y 4 con respecto al carbono de la función éster, por ello de acuerdo con la nomenclatura IUPAQ son denominados 3-piperidincarboxilato de etilo y 4-piperidincarboxilato de etilo (Carey y Sundberg, 2007); las piperidinas sustituidas en las posiciones 3 y 4 pueden ser obtenidas a partir de la reacción de sustitución nucleofílica entre el glutaraldehído (sustituído en las posiciones 2 o 3) y el amoníaco o una amina primaria, seguida de un proceso de hidrogenación, estas síntesis son muy selectivas y presentan altos rendimientos (Risi et al., 2008; Micalizio y Chen, 2008;Jiang et al., 2011)

El nipecotato de etilo e isonipecotato de etilo han sido muy poco estudiados, sin embargo algunos derivados piperidínicos han demostrado tener importancia a nivel industrial en diversas reacciones químicas (Goka et al., 2004), además de su uso en la elaboración de productos farmacológicos (Vargas y Kouznetsov, 2007; Dantzman et al., 2012), para el tratamiento de las adicciones principalmente, también como antidepresivos (Revanasiddappa et al., 2012) y anticancerígenos (Mostafa et al., 2013). En la literatura científica se han encontrado escasos estudios de termólisis de ésteres con anillos nitrogenados, como es el caso del picolinato de etilo y 1-metilpipecolinato de etilo, el primero con anillo piridínico que se descompone térmicamente formando el ácido respectivo y este se descarboxila hasta piperidina; el segundo posee un ciclo nitrogenado sustituido, ambas descomposiciones térmicas fueron realizadas en fase gaseosa y describen un mecanismo cíclico unimolecular y de primer orden (Lafont et al., 2003), también se han reportado las termólisis de ésteres con otros grupos funcionales en las cuales se proponen mecanismos similares, (House, 2007) donde las descomposiciones térmicas ocurren en el grupo éster y no en las cadenas adyacentes (Zapata et al., 2007; Rodríguez et al., 2009).

El estudio cinético de la descomposición térmica del nipecotato de etilo e isonipecotato de etilo no han sido reportados en la literatura, razón por la cual con este trabajo se pretende realizar un aporte importante a la comunidad académica y científica, cuyo objetivo consistió en realizar la termólisis de estos compuestos en el rango de temperaturas entre $(523,15-563,15) \mathrm{K}$ con diferencia de diez grados, con el fin de evaluar el comportamiento de las constantes de velocidad con respecto a la temperatura y la influencia de la cercanía del átomo de nitrógeno presente en el ciclo con respecto al grupo éster sobre la velocidad de reacción, verificar cual de los dos compuestos se degrada más rápidamente, encontrar el orden de cada reacción, calcular los parámetros de activación y proponer un posible mecanismo de descomposición térmica.

\section{METODOLOGÍA}

Los compuestos nipecotato e isonipecotato de etilo fueron adquiridos de la empresa Alfa Aesar, con purezas del $97 \%$ y $98 \%$ respectivamente, los cuales fueron verificados utilizando un equipo de cromatografía de gases, estando en buen acuerdo. Para realizar las reacciones de descomposición térmica en fase gaseosa, se empleó un reactor de escala semimicro dotado de un horno cilíndrico que posee en la parte superior veinte perforaciones (cada una de $5.5 \mathrm{~cm}$ de diámetro y $8.0 \mathrm{~cm}$ de longitud), donde se insertan la ampolletas de vidrio (con diámetro interno de $2 \mathrm{~mm}$ y longitud de $4 \mathrm{~cm}$ ) con la muestra a termolizar; este sistema es regulado con un sensor de temperatura termocoupla tipo $\mathrm{K}$, cuyo intervalo de calentamiento puede variar entre $(273,15-1073,15) \mathrm{K}$, con incertidumbre de $\pm 1 \mathrm{~K}$.

El procedimiento realizado en la termólisis del nipecotato de etilo consistió en agregar $20 \mu \mathrm{L}$ del compuesto $(0.02 \mathrm{~mL})$ en cada ampolleta hasta completar seis por serie, las cuales fueron cerradas en ausencia de aire utilizando un soplete, posteriormente se insertaron en los orificios del horno del reactor, previamente calibrado a la temperatura de $523,15 \mathrm{~K}$, luego a tiempos constantes se fueron sacando las ampolletas y se sumergieron en un baño de hielo para detener la reacción, en seguida se inyectó la muestra en un cromatógrafo de gases acoplado a espectrometría de masas; para obtener los valores de las áreas de descomposición del reactivo y de formación del producto con respecto al tiempo. Este procedimiento se realizó por triplicado a las temperaturas de $(523,15 ; 533,15 ; 543,15 ; 553,15$ y 563,15$) \mathrm{K}$; para ambos compuestos nipecotato e isonipecotato de etilo (Lafont et al., 2009; Milanés et al., 2012; Ensuncho et al., 2011).

Las determinaciones cuantitativas de las áreas fueron realizadas utilizando un equipo de cromatografía de gases marca Perkin Elmer, modelo Agilent System XL, con detector FID, se utilizó una columna AB-5MS (0,25 $\mathrm{mm}$ de diámetro interno $\times 30 \mathrm{~m}$ longitud nominal $\times 0,25 \mu \mathrm{m}$ espesor de la película) cuya fase estacionaria fue $95 \%$ de dimetilpolisiloxano y $5 \%$ de difenilo, se programó una rampa de calentamiento que inició con 120C y aumentó $10 \mathrm{C}$ por minuto hasta alcanzar la temperatura de 190C, la temperatura del inyector fue de 190C y del detector FID de 200C, se empleó el método cuantitativo del patrón interno (metanol). 
Para hallar las constantes de velocidad en las reacciones de termólisis del nipecotato de etilo e isonipecotato de etilo a diferentes temperaturas, se supone una ecuación de primer orden $(A \rightarrow P)$, donde $\mathrm{A}$ es el reactivo y $\mathrm{P}$ los productos, se supone que la concentración inicial de $\mathrm{A}$ a $\mathrm{t}=0$ es igual a $a$ y $\mathrm{x}$ representa el cambio en la concentración a medida que la reacción avanza. Cuando ha transcurrido un tiempo t desde el inicio de la reacción la concentración de $\mathrm{A}$ ha disminuido hasta $(a-x)$ y $\mathrm{P}$ ha aumentado a x; para lo cual es representado mediante la expresión (1), que es integrada hasta obtener la ecuación (5) que relaciona las concentraciones de los reactivos con el tiempo. Para obtener las constantes de velocidad a cada temperatura de los compuestos en estudio se graficó $\ln (a-x)$ vs t cuya pendiente negativa es igual a la constante de velocidad, y el intercepto corresponde a la concentración inicial del reactivo $\ln a$ (Lafont et al., 2013; Logan, 2000).

$-\frac{d[a-x]}{d t}=\frac{d x}{d t} k[a-x]$

Separando las variables $\mathrm{x}$ y $\mathrm{t}$ y reordenando la ecuación se tiene:

$$
\frac{d x}{a-x}=k d t
$$

Integrando ambos miembros se obtiene:

$$
\begin{aligned}
& \int \frac{d x}{a-x}=\int k d t \quad ; \quad-\int-\frac{d x}{a-x}=k \int d t \\
& -\ln (a-x)=k t+C
\end{aligned}
$$

Cuando $\mathrm{t}=0 ; \mathrm{x}=0 \quad$ entonces $C=-\ln a$

$\ln a-\ln (a-x)=k t$

A partir de la forma logarítmica de la ecuación de Arrhenius (6) que relaciona los valores de la constante de velocidad con la temperatura, se obtuvieron los valores de las energías de activación (Ea), para cada reacción de descomposición térmica, mediante la representación gráfica de Ink vs $1 / T(K)$ cuya pendiente corresponde a $-\mathrm{Ea} / \mathrm{R}$, siendo $\mathrm{R}$ la constante de los gases. De la ecuación de Eyring (7) que describe la relación entre la constante de velocidad con la temperatura se obtuvieron los parámetros de activación, donde $\Delta \mathrm{H}^{\neq}$es el cambio en la entalpía de activación, $\mathrm{T}$ la temperatura absoluta $(\mathrm{K}), \mathrm{k}_{\beta}$ representa la constante de Boltzman, $\mathrm{h}$ la constante de Planck y $\Delta \mathrm{S}^{\neq}$el cambio de entropía en el estado de transición (House, 2007; Logan, 2000).

$$
\begin{aligned}
& \operatorname{Lnk}=-\frac{E_{a}}{R T}+\operatorname{Ln} A \\
& \operatorname{Ln} \frac{k}{T}=\frac{-\Delta H^{\neq}}{R} \frac{1}{T}+\operatorname{Ln} \frac{k_{\beta}}{h}+\frac{\Delta S^{\neq}}{R}
\end{aligned}
$$

También se simularon teóricamente los estados de transición posibles de la termólisis de ambos compuestos empleando el método computacional DFT, una base 6-31G* y el funcional B3LYP implementados en el programa Gaussian-98 (Frich et al., 1998) para el nipecotato e isonipecotato de etilo respectivamente; con el fin de comparar las energías de activación. 


\section{RESULTADOS Y DISCUSIÓN}

En las Fig. 1 y 2 se observan las constantes de velocidad obtenidas de las pendientes de los gráficos de $\ln (a-x)$ vs t para el nipecotato de etilo e isonipecotato de etilo a 523,15K respectivamente, los cuales se ajustaron a una tendencia lineal con pendiente negativa, este comportamiento también fue encontrado a las temperaturas de $(533,15 ; 543,15 ; 553,15$ y 563,15$) \mathrm{K}$, como se observa en los valores de $r^{2}$ presentados en la Tabla 1; demostrando que las descomposiciones térmicas de ambos compuestos obedecen la Ley cinética de primer orden.
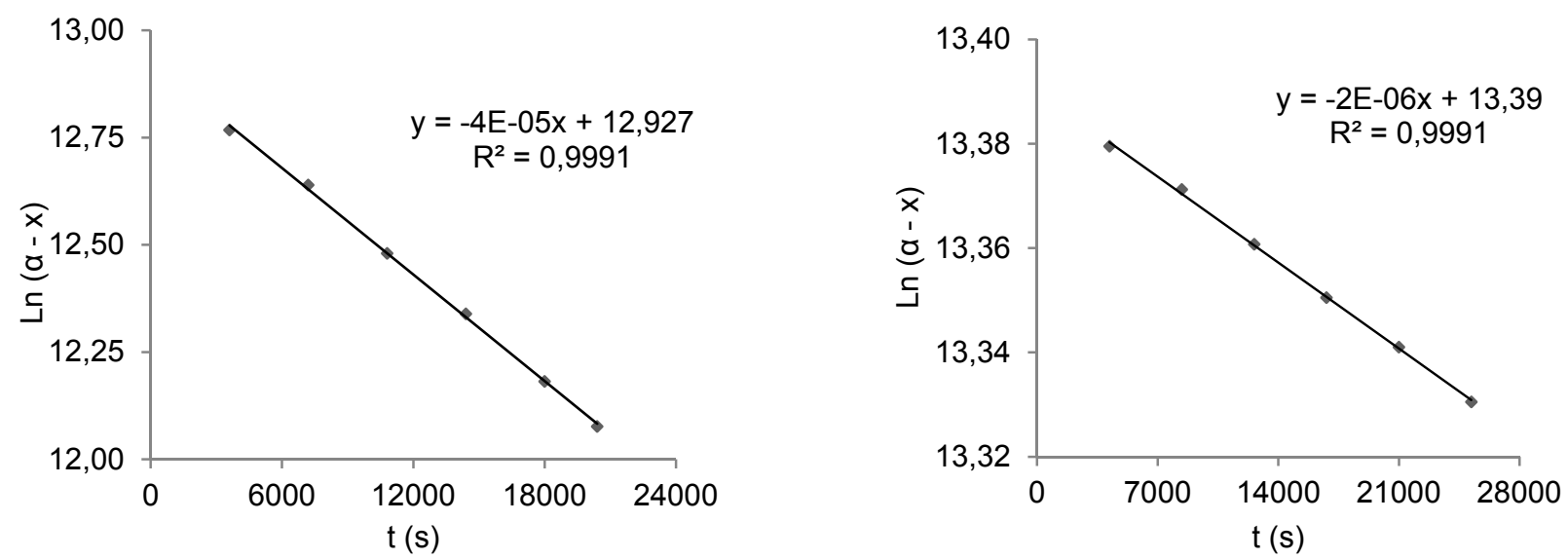

Fig. 1: Obtención de constantes de velocidad para el nipecotato de etilo e isonipecotato de etilo a 523,15 K

De la Tabla 1 se evidencia que el isonipecotato de etilo presentó constantes de velocidad menores comparadas con las del nipecotato de etilo a las mismas temperaturas de estudio; también se observó para ambos compuestos, el aumento de la velocidad de termólisis con el incremento en la temperatura, estos resultados estan acorde con la expresión de Arrhenius (2) (Carey y Sundberg, 2007), la cual es representada en la Fig. 2 donde se evidencia una tendencia lineal para el nipecotato de etilo e isonipecotato de etilo con valores de $R^{2}$ de 0.9991 y 0.9995 respectivamente.

Tabla 1: Constantes de velocidad de los compuestos en estudio a diferentes temperaturas

\begin{tabular}{rcccc}
\hline Compuesto & $\mathrm{k}\left(\mathrm{s}^{-1}\right)$ & $\mathrm{T}(\mathrm{K})$ & $1000 / \mathrm{T}\left(\mathrm{K}^{-1}\right)$ & $\mathrm{R}^{2}$ \\
\hline \multirow{3}{*}{ Nipecotato de etilo } & $4,14 \mathrm{E}-05 \pm 0,04$ & 523,15 & 1,91 & 0,9991 \\
& $8,13 \mathrm{E}-05 \pm 0,70$ & 533,15 & 1,88 & 0,9995 \\
& $1,81 \mathrm{E}-04 \pm 0,42$ & 543,15 & 1,84 & 0,9992 \\
& $3,54 \mathrm{E}-04 \pm 0,55$ & 553,15 & 1,81 & 0,9996 \\
& $6,66 \mathrm{E}-04 \pm 0,20$ & 563,15 & 1,78 & 0,9991 \\
\hline \multirow{3}{*}{ Isonipecotato de etilo } & $2,23 \mathrm{E}-06 \pm 0,12$ & 523,15 & 1,91 & 0,9991 \\
& $4,65 \mathrm{E}-06 \pm 0,53$ & 533,15 & 1,88 & 0,9992 \\
& $1,00 \mathrm{E}-05 \pm 0,35$ & 543,15 & 1,84 & 0,9994 \\
& $2,17 \mathrm{E}-05 \pm 0,60$ & 553,15 & 1,81 & 0,9998 \\
& $4,49 \mathrm{E}-05 \pm 0,10$ & 563,15 & 1,78 & 0,9997 \\
\hline
\end{tabular}

Con base en los valores de las pendientes de los gráficos de Arrhenius (Fig. 2) para ambos compuestos se hallaron las energías de activación (Ea) para el nipecotato de etilo $\left(41,14 \pm 0.15 \mathrm{Kcal}^{\mathrm{mol}}{ }^{-1}\right)$ y para el isonipecotato de etilo $\left(44,16 \pm 0.38 \mathrm{Kcal}_{\mathrm{mol}}{ }^{-1}\right)$ reportados en la Tabla 2. De acuerdo con estos datos el segundo compuesto presentó una energía de activación mayor, lo cual está relacionado con una velocidad de descomposición térmica más lenta, como se evidenció con los valores de las constantes de velocidad menores (Tabla 1) comparado con el nipecotato de etilo a las mismas temperaturas en estudio; de igual forma las energías de activación de ambos compuestos fueron muy parecidos a sus entalpías de activación correspondientes, estando acorde con la expresión Ea $=\Delta \mathrm{H}^{\neq}+n R T$. 


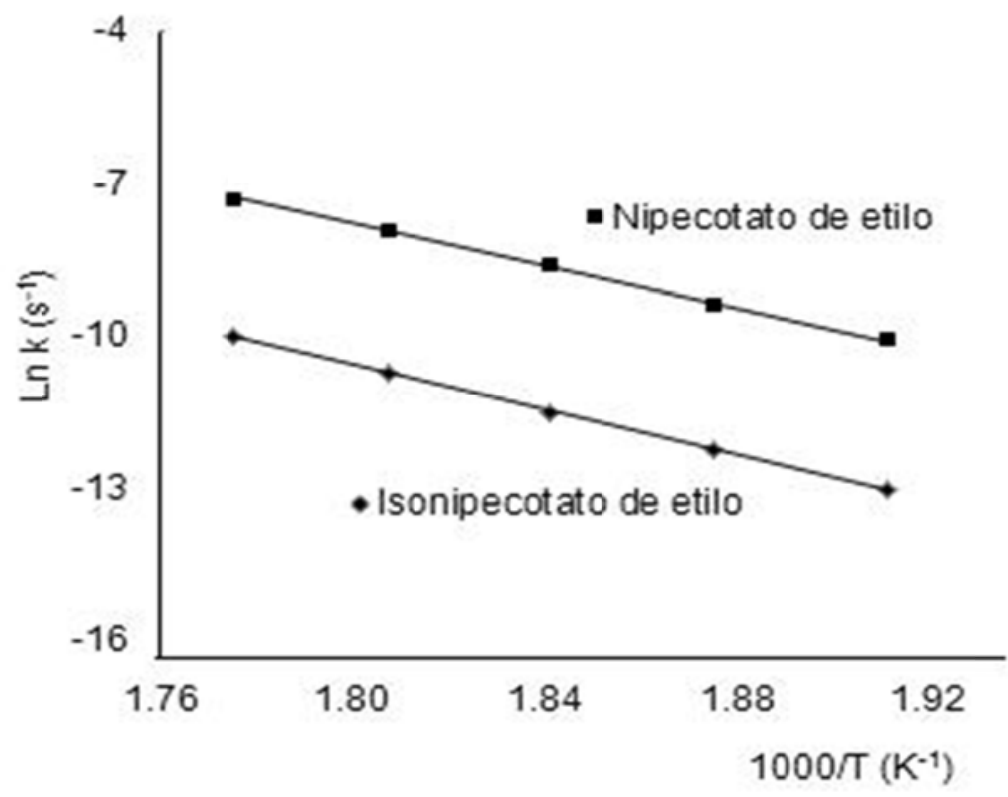

Fig. 2: Gráfico de Arrhenius para el nipecotato de etilo e isonipecotato de etilo a 543,15 K

A partir de la Fig. 2 también se obtuvieron los valores del factor preexponencial (A) para el nipecotato $\left(6,315 \mathrm{E} 12 \pm 0.54 \mathrm{~s}^{-1}\right)$ e isonipecotato de etilo $\left(6,073 \mathrm{E}-12 \pm 0.72 \mathrm{~s}^{-1}\right)$, los cuales estuvieron en el rango de $\left(10^{11}-10^{13}\right) \mathrm{s}^{-1}$ reportado en la literatura para reacciones de primer orden (Zapata et al., 2007; Julio et al., 2007).

Los datos teóricos reportados se han obtenido empleando como método de cálculo DFT usando una base 6-31G* y el funcional B3LYP implementados en el programa Gaussian-98 (Frisch et al., 1998). Todas las estructuras de posible punto crítico fueron optimizadas con el fin de comparar las energías de activación para el nipecotato e isonipecotato de etilo respectivamente. Se simularon los estados de transición para proponer el más favorable a través del cual transcurren las reacciones de termólisis, representados en la Fig.3; para formar el ácido piperidincarboxílico correspondiente; es conocido que la función éster posee dos oxígenos con electrones libres, que pueden actuar como nucleófilo.

Sin embargo no se puede desconocer el carácter básico de las aminas, por ello se analizó la posibilidad de que el nitrógeno participara del estado de transición, en lugar del oxígeno, los dos primeros estados de transición involucran el átomo de nitrógeno para el nipecotato de etilo e isonipecotato de etilo respectivamente y los dos últimos el átomo de oxígeno de la función éster; de este análisis se encontró que los estados de transición que incluyen el átomo de nitrógeno utilizando la misma metodología, presentaron mayor energía de activación $\left(625,312 \mathrm{Kcal} \mathrm{mol}^{-1}\right.$ para el nipecotato de etilo y $629,034 \mathrm{Kcal}^{\mathrm{mol}}{ }^{-1}$ para el isonipecotato de etilo ) comparados con los otros dos estados de transición que involucran el átomo de oxígeno, con energías de activación más bajas $\left(47,754 \mathrm{Kcal}^{\mathrm{mol}}{ }^{-1}\right.$ para el nipecotato de etilo y 50,675 Kcal.mol ${ }^{-1}$ para el isonipecotato de etilo) haciéndolos más factibles; estos valores teóricos están acorde con los datos experimentales $(41,14 \pm 0,15$ y 44,16 $\pm 0,38) \mathrm{Kcal}_{\text {. mol }}{ }^{-1}$ respectivamente; por lo anterior se puede decir que la posición del átomo de $\mathrm{N}$ no influye en la etapa determinante de la velocidad que corresponde a la formación inicial del ácido nipecotico e isonipecotico con eliminación de la molécula de etileno.

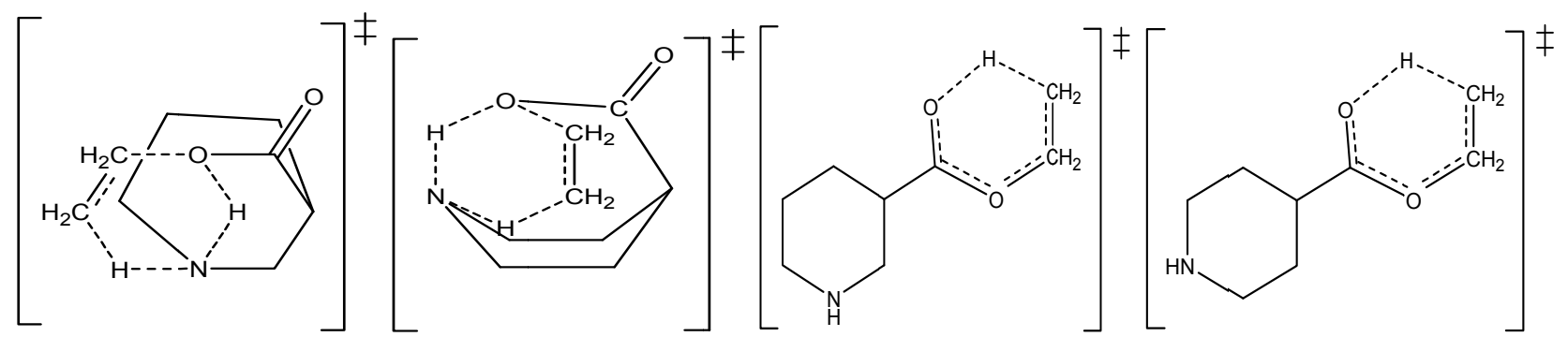

Fig. 3: Estados de transición del nipecotato e isonipecotato de etilo con participación de los átomos de nitrógeno y oxígeno respectivamente 
Las entalpías de activación $\left(\Delta \mathrm{H}^{\ddagger}\right)$ encontradas en la Tabla 2, para el nipecotato de etilo $(40,06 \pm 0,61$ Kcal.mol $\left.{ }^{-1}\right)$ e isonipecotato $\left(43,08 \pm 0,50 \mathrm{Kcal}_{\mathrm{mol}}{ }^{-1}\right)$ fueron positivas, mostrando procesos endotérmicos y fue mayor para el isonipecotato, lo cual sugiere que la termólisis de este compuesto presentó mayor barrera energética y por ende menor velocidad de la reacción de descomposición térmica. Las energías libres de activación $\left(\Delta \mathrm{G}^{\mp}\right)$ encontradas para el nipecotato de etilo $\left(41,76 \pm 0,90 \mathrm{Kcal}^{\mathrm{mol}}{ }^{-1}\right)$ e isonipecotato de etilo $\left(44,83 \pm 0,60 \mathrm{Kcal}^{\mathrm{mol}}{ }^{-1}\right)$, fueron positivas describiendo un proceso no espontaneo, cuyos valores son muy cercanos debido a que son isómeros, sin embargo se observó un leve incremento en el segundo compuesto lo cual está acorde con las menores constantes de velocidad, haciendo más lenta la reacción.

La entropía de activación $\left(\Delta S^{\ddagger}\right)$ puede interpretarse como el grado de desorden de un sistema, los valores de la entropía de activación para ambos compuestos fueron negativos y similares para el nipecotato de etilo $\left(-3,14 \pm 0.42\right.$ cal.mol $\left.{ }^{-1} \mathrm{~K}^{-1}\right)$ e isonipecotato de etilo $\left(-3,22 \pm 0.26 \mathrm{cal}^{\mathrm{mol}} \mathrm{m}^{-1} \mathrm{~K}^{-1}\right)$, lo cual sugiere un estado de transición más ordenado y con menos grados de libertad con relación a los reactivos.

Tabla 2: Parámetros de activación para los compuestos en estudio calculados a 543,15K

\begin{tabular}{lll}
\hline Parámetros de Activación & Nipecotato e etilo & Isonipecotato de etilo \\
\hline $\mathrm{k}\left(\mathrm{s}^{-}\right)$ & $1,81 \mathrm{E}-04 \pm 0,42$ & $1,00 \mathrm{E}-05 \pm 0,35$ \\
$\mathrm{Ea}\left(\mathrm{Kcal} \cdot \mathrm{mol}^{-1}\right)$ & $41,14 \pm 0,15$ & $44,16 \pm 0,38$ \\
$\mathrm{~A}\left(\mathrm{~s}^{-1}\right)$ & $6,31 \mathrm{E}-12 \pm 0,54$ & $6,07 \mathrm{E}-12 \pm 0,72$ \\
$\Delta \mathrm{H}^{\neq}\left(\mathrm{Kcal} . \mathrm{mol}^{-1}\right)$ & $40,06 \pm 0,61$ & $43,08 \pm 0,50$ \\
$\Delta \mathrm{S}^{\ddagger}\left(\mathrm{cal} . \mathrm{mol}^{-1} \mathrm{~K}^{-1}\right)$ & $-3,14 \pm 0,42$ & $-3,22 \pm 0,26$ \\
$\Delta \mathrm{G}^{\neq}\left(\mathrm{Kcal}^{\mathrm{mol}}{ }^{-1}\right)$ & $41,76 \pm 0,90$ & $44,83 \pm 0,60$ \\
\hline
\end{tabular}

Los productos de la descomposición térmica del nipecotato de etilo con un $80 \%$ de avance en la reacción a las temperaturas de trabajo fueron identificados como ácido nipecótico (ácido 3-piperidincarboxílico); piperidina y etileno, mediante cromatografía de gases acoplada a espectrometría de masas, con un $99 \%$ de correspondencia con las bases de datos de los estándares de referencia NIST disponibles en el equipo; de igual forma se determinaron los productos de termólisis del isonipecotato de etilo con un avance del $85 \%$ de la reacción a las temperaturas ensayadas y los productos obtenidos fueron el ácido isonipecótico (ácido 4piperidincarboxílico), piperidina y etileno, los cuales presentaron una correspondencia del $99 \%$ con la base de datos de la NIST. De acuerdo con los análisis teóricos la etapa determinante de la velocidad para cada termólisis corresponde a la formación del ácido nipecotico e isonipecotico que posteriormente es descarboxilado debido a la intervención del átomo de nitrógeno en la desprotonación del grupo ácido para formar la amina cíclica correspondiente con eliminación de dióxido de carbono.

En la literatura (Lafont et al., 2003) se ha reportado estudios sobre la termólisis de compuestos que poseen un ciclo nitrogenado unido a la función ácido similar a uno de los productos de los compuestos en estudio, estos se descarboxilan formando dióxido a de carbono y la amina cíclica correspondiente, mecanismo donde se observa la intervención activa del nitrógeno al sustraer el hidrogeno del grupo funcional ácido; como se aprecia en la Fig. 4.
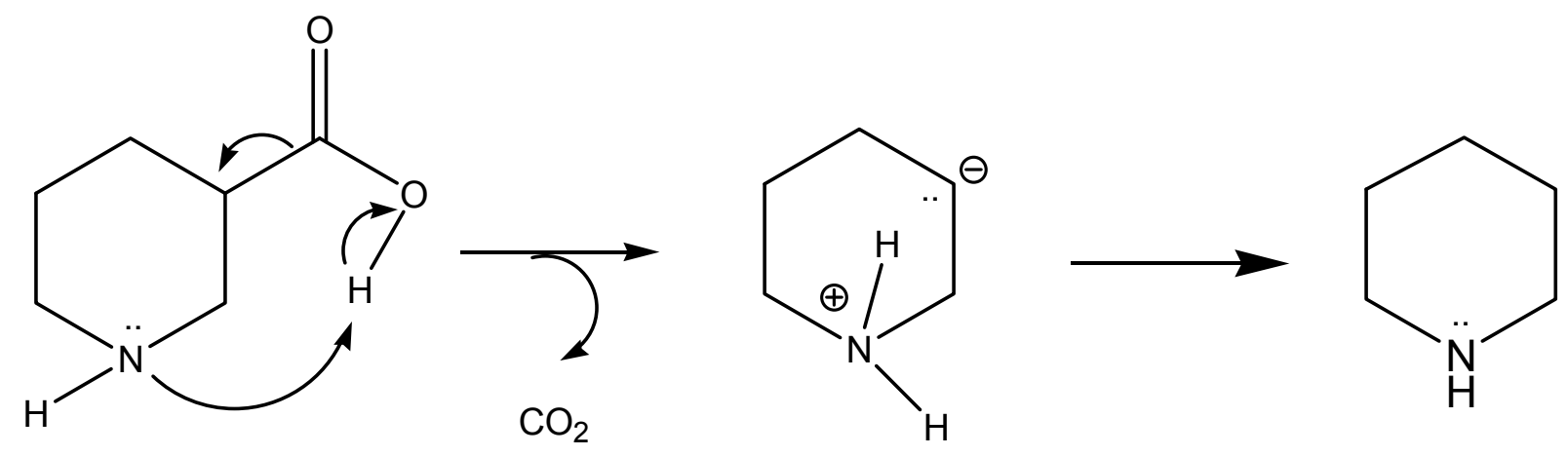

Fig.4: Descarboxilación del ácido 3-piperidincarboxílico 
De acuerdo con los productos identificados en las termólisis de ambos compuestos, los valores de los parámetros de activación y las energías de los estados de transición, se propone un mecanismo similar de descomposición térmica, que transcurre a través de un estado de transición cíclico de seis miembros, semipolar y concertado, el cual produce etileno y ácido nipecótico (o isonipecótico) que luego sufre una descarboxilación y es transformado en piperidina y dióxido de carbono, este mecanismo se muestra en la Fig. 5.

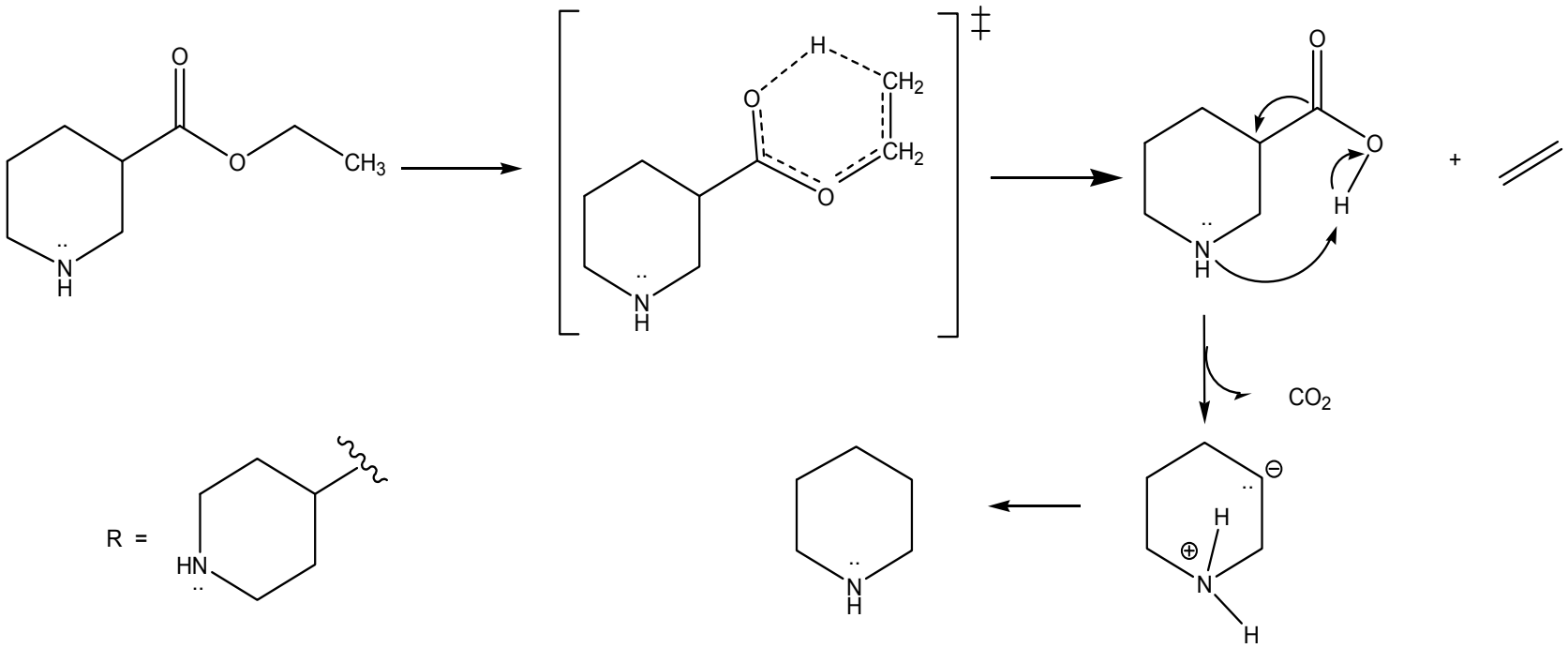

Fig. 5: Mecanismo de termólisis propuesto para los compuestos nipecotato de etilo e isonipecotato de etilo

\section{CONCLUSIONES}

Después de analizar y discutir los resultados de las reacciones de termólisis de los compuestos nipecotato e isonipecotato de etilo a las cinco temperaturas en estudio, se puede concluir que ambas descomposiciones térmicas siguen la Ley cinética de primer orden. Se encontró que el proceso de degradación frente a los cambios de temperatura fue más rápido para el nipecotato de etilo, lo cual se reflejó en las constantes de velocidad mayores para cada temperatura y estuvo acorde con el menor valor en la energía de activación para este compuesto, al igual que con los cálculos teóricos donde se comprobó que el átomo de nitrógeno inmerso en una posible estructura de transición genera una Ea más alta y por tanto menos estable, por lo cual se puede demostrar que este no influye en la velocidad de la reacción, sino que participa en la desprotonación del grupo funcional ácido. De acuerdo con los productos identificados, los valores de energías de activación teóricos y experimentales y demás parámetros en el estado de transición, se propuso un mecanismo que transcurre a través de un estado de transición cíclico de seis miembros, que produce el ácido carboxílico correspondiente, el cual se descarboxila generando los productos finales de piperidina, etileno y dióxido de carbono.

\section{AGRADECIMIENTOS}

A la Universidad de Córdoba por financiar este proyecto.

\section{REFERENCIAS}

Carey, F.A.; R. J. Sundberg, Advanced Organic Chemistry Part A: Structure and Mechanisms, $5^{\mathrm{a}}$ edición, 682-684, Springer Science, New York, EUA (2007).

Dantzman, C.L., y otros catorce autores, 4-Piperidin-4-ylidenemethyl-benzamides as $d$-opioid receptor agonists for CNS indications: Identifying clinical candidates, Bioorganic \& Medicinal Chemistry Letters: 22 1174-1178 (2012).

Ensuncho, A., N. Milanés., J. Robles, Estudio Experimental y Computacional de la Cinética de Termólisis del 2,5-Hexanodiol, Información Tecnológica: 22 (6), 87-94 (2011).

Frisch, M. J., y otros cincuenta y nueve autores, Gaussian 98, Gaussian, Inc., Pittsburgh, PA, (1998).

Goka, V. N, y otros cinco autores, Syntheses and GABA uptake properties of 6-ether- and 6-enol ethersubstituted nipecotic acids, European Journal of Medicinal Chemistry: 39, 633-638 (2004). 
House, J.E., Principles of Chemical Kinetics, $2^{\text {a }}$ Edición,111-119, Academic Press Elsevier, San Diego, EUA (2007).

Jiang, H., J. Xiaochen., Y. Li., Z. Chen., A. Wang., Palladium-assisted multicomponent cyclization of aromatic aldehydes, arylamines and terminal olefins under molecular oxygen: an assemblyof 1,4dihydropyridines, Org. Biomol. Chem: 9, 5358-5361 (2011).

Julio, L., J. Lafont., J. Quijano., G. Chuchani, The unimolecular Elimination Kinetics of benzaldoxime in the gas phase, International Journal of Chemical Kinetics: 39, 145-147 (2007).

Lafont, J.J.,y otros seis autores, Mechanism of elimination of amino acid derivatives in the gas phase. Pyrolysis kinetics of ethyl picolinate, ethyl-1-methylpipecolinate and picolinic acid, Journal of Physical Organic Chemistry: 16, 84-88 (2003).

Lafont, J.J., M. S. Páez., A. Alvis, Estudio Cinético de la Descomposición Térmica de Tres Derivados del Pirano $\left(\mathrm{C}_{5} \mathrm{H}_{6} \mathrm{O}\right)$, Información Tecnológica: 20(5), 39-46 (2009).

Lafont, J.J., Y. C. Torres., E. Lans, Termólisis de la 4-Hidroxi-3-Metil-2- Butanona y Análisis del Efecto de Solventes a Diferentes Temperaturas, Información Tecnológica: 24(3), (2013) en prensa.

Logan, S. R., Fundamentos de cinètica quìmica, $1^{a}$ Ediciòn, 1-62, Addison Wesley, Madrid, España (2000).

Micalizio, G.C., Chen, M.Z., Convergent Synthesis of Piperidines by the Union of Conjugated Alkynes with Imines: A Unique Regioselective Bond Construction for Heterocycle Synthesis, Bioorg. Med. Chem: 16, 601635 (2008).

Milanés, N., A. Ensuncho., J. Robles, Obtención de Dímeros por Termólisis de 1,2-Propanodiol, Información Tecnológica: 23 (5), 3-12 (2012).

Mostafa, S.I., y otros cinco autores, New zinc(II), palladium(II) and platinum(II) complexes of dl-piperidine-2carboxylic acid; X-ray crystal structure of trans- $\left[\mathrm{Zn}_{2}(\mu-\mathrm{Ca})_{2}(\mathrm{Hpa})_{2} \mathrm{Cl}_{6}\right]$ and anticancer activity of some complexes, Journal of Molecular Structure: 1036,161-167 (2013).

Revanasiddappa, H. D., M.K. Prashan., K.M., L B. Veeresh, Synthesis, characterization, antidepressant and antioxidant activity of novel piperamides bearing piperidine and piperazine analogues, Bioorganic \& Medicinal Chemistry Letters: 22 (23), 7065-7070 (2012).

Risi, C., y otros cinco autores, Recent advances in the stereoselective synthesis of trans-3,4-disubstitutedpiperidines: applications to (-)-paroxetine, Tetrahedron Asymmetry: 19, 131-155 (2008).

Vargas, L.Y., y V.V, Kouznetsov, 4-aminopiperidinas y espiro-4-piperidinas: Importancia farmacológica y esfuerzos Sintéticos, Universitas Scientiarum: 12 (2), 23-45 (2007).

Zapata, E., J. Gaviria., J. Quijano, Thermal descomposition of methyl $\beta$-Hydroxyesters in m-xylene solution, International Journal of Chemical kinetics: 39 (2), 92-96 (2007). 